\title{
THE HERMITE-SYLVESTER CRITERION FOR REAL-ROOTED POLYNOMIALS
}

\author{
MELVYN B. NATHANSON
}

\begin{abstract}
A polynomial is real-rooted if all of its roots are real. This note gives a simple proof of the Hermite-Sylvester theorem that a polynomial $f(x) \in$ $\mathbf{R}[x]$ is real-rooted if and only if an associated quadratic form is positive semidefinite.
\end{abstract}

A polynomial is real-rooted if all of its roots are real. Hermite and Sylvester [1] proved that a polynomial $f(x) \in \mathbf{R}[x]$ is real-rooted if and only if a certain quadratic form is positive semidefinite. Here is a short proof of the Hermite-Sylvester theorem that uses only Lagrange interpolation.

The quadratic form associated with a real $n \times n$ matrix $H=\left(h_{i, j}\right)$ is

$$
Q=Q\left(x_{1}, \ldots, x_{n}\right)=\sum_{i=1}^{n} \sum_{j=1}^{n} h_{i, j} x_{i} x_{j} .
$$

This form is positive semidefinite if $Q\left(x_{1}, \ldots, x_{n}\right) \geq 0$ for all vectors $\left(\begin{array}{c}x_{1} \\ \vdots \\ x_{n}\end{array}\right) \in \mathbf{R}^{n}$.

Let $f(x)$ be a polynomial of degree $n$, and let $\lambda_{1}, \ldots, \lambda_{n}$ be the (not necessarily distinct) roots of $f(x)$. Define the $k$ th power sum

$$
m_{k}=m_{k}\left(\lambda_{1}, \ldots, \lambda_{n}\right)=\sum_{\ell=1}^{n} \lambda_{\ell}^{k} .
$$

Newton's identities [2] enable the efficient computation of the numbers $m_{k}$ from the coefficients of $f(x)$. The Hermite matrix constructed from the polynomial $f(x)$ is the $n \times n$ matrix

$$
H_{f}=\left(\begin{array}{ccccc}
m_{0} & m_{1} & m_{2} & \cdots & m_{n-1} \\
m_{1} & m_{2} & m_{3} & \cdots & m_{n} \\
m_{2} & m_{3} & m_{4} & \cdots & m_{n+1} \\
\vdots & & & & \vdots \\
m_{n-1} & m_{n} & m_{n+1} & \cdots & m_{2 n-2}
\end{array}\right)=\left(h_{i, j}\right)
$$

where $h_{i, j}=m_{i+j-2}$ for all $i, j \in\{1, \ldots, n\}$.

Let $\bar{\lambda}$ denote the complex conjugate of the complex number $\lambda$.

Date: November 26, 2019.

2010 Mathematics Subject Classification. 05C31, 11C08, 15A15, 65H04.

Key words and phrases. Hermite-Sylvester criterion, real-rooted polynomials, Newton's identities, Lagrange interpolation.

Supported in part by a grant from the PSC-CUNY Research Award Program. 
Lemma 1. Let $\Lambda$ be a nonempty finite set of $r$ complex numbers that is closed under complex conjugation, that is,

$$
\Lambda=\{\bar{\lambda}: \lambda \in \Lambda\} .
$$

Let

$$
p(t)=\sum_{j=0}^{r-1} c_{j} t^{j} \in \mathbf{C}[t]
$$

be a polynomial of degree at most $r-1$. If

$$
p(\bar{\lambda})=\overline{p(\lambda)}
$$

for all $\lambda \in \Lambda$, then $p(t) \in \mathbf{R}[t]$.

Proof. We use the fact that if polynomials $p(t) \in \mathbf{C}[t]$ and $q(t) \in \mathbf{C}[t]$ have degrees at most $r-1$, and if $p(\lambda)=q(\lambda)$ for all $\lambda$ in a set of size $r$, then $p(t)=q(t)$. Let

$$
q(t)=\sum_{j=0}^{r-1} \overline{c_{j}} t^{j}
$$

For all $\lambda \in \mathbf{C}$, we have $\overline{\bar{\lambda}}=\lambda$. For all $\lambda \in \Lambda$, we have $\bar{\lambda} \in \Lambda$, and so

$$
p(\lambda)=p(\overline{\bar{\lambda}})=\overline{p(\bar{\lambda})}=\overline{\sum_{j=0}^{r-1} c_{j} \bar{\lambda}^{j}}=\sum_{j=0}^{r-1} \overline{c_{j}} \overline{\bar{\lambda}}^{j}=\sum_{j=0}^{r-1} \overline{c_{j}} \lambda^{j}=q(\lambda) .
$$

Therefore, $p(t)=q(t)$, and so, for all $j \in\{0,1, \ldots, r-1\}$, we have $c_{j}=\overline{c_{j}} \in \mathbf{R}$ and $p(t) \in \mathbf{R}[t]$. This completes the proof.

Lemma 2. Let $\Lambda=\left\{\lambda_{1}, \lambda_{2}, \lambda_{3}, \ldots, \lambda_{r}\right\}$ be a nonempty finite set of $r$ complex numbers that is closed under complex conjugation. Suppose that $\lambda_{1} \in \Lambda$ is not real, and that $\lambda_{2}=\overline{\lambda_{1}} \in \Lambda$. There exists a polynomial $p(t) \in \mathbf{R}[t]$ of degree at most $r-1$ such that

$$
p\left(\lambda_{1}\right)=i, \quad p\left(\lambda_{2}\right)=-i
$$

and

$$
p\left(\lambda_{j}\right)=0 \quad \text { for all } j \in\{3,4, \ldots, r\} .
$$

Proof. By Lagrange interpolation, there exists a polynomial $p(t) \in \mathbf{C}[t]$ of degree at most $r-1$ that satisfies (2) and (3). We have

$$
p\left(\overline{\lambda_{1}}\right)=p\left(\lambda_{2}\right)=-i=\bar{i}=\overline{p\left(\lambda_{1}\right)} .
$$

and

$$
p\left(\overline{\lambda_{2}}\right)=p\left(\lambda_{1}\right)=i=\overline{-i}=\overline{p\left(\lambda_{2}\right)} .
$$

Also, $p\left(\overline{\lambda_{j}}\right)=0=\overline{p\left(\lambda_{j}\right)}$ for all $j \in\{3,4, \ldots, r\}$. It follows from Lemma 1 that $p(t) \in \mathbf{R}[t]$. This completes the proof.

Theorem 1 (Hermite-Sylvester). The nonconstant polynomial $f(x) \in \mathbf{R}[x]$ is realrooted if and only if the quadratic form $Q_{f}$ constructed from the Hermite matrix $H_{f}$ is positive semidefinite. 
Proof. Let $f(x) \in \mathbf{R}[x]$ be a polynomial of degree $n \geq 1$, and let $\Lambda$ be the set of distinct roots of $f(x)$. We have $|\Lambda|=r \leq n$. Let $\Lambda=\left\{\lambda_{1}, \lambda_{2}, \ldots, \lambda_{r}\right\}$. Because $f(x)$ has real coefficients, the set $\Lambda$ is closed under conjugation.

Extend the sequence $\lambda_{1}, \lambda_{2}, \ldots, \lambda_{r}$ of $r$ distinct roots of $f(x)$ to the sequence $\lambda_{1}, \ldots, \lambda_{r}, \lambda_{r+1}, \ldots, \lambda_{n}$ of $n$ roots of $f(x)$ with multiplicity. Thus, for $j \in\{1, \ldots, r\}$, the root $\lambda_{j}$ with multiplicity $\mu_{j}$ appears $\mu_{j}$ times in this sequence.

Let $H_{f}=\left(h_{i, j}\right)$ be the Hermite matrix constructed from the polynomial $f(x)$, and let $Q_{f}$ be the quadratic form constructed from $H_{f}$. For $\left(\begin{array}{c}x_{1} \\ \vdots \\ x_{n}\end{array}\right) \in \mathbf{R}^{n}$, we have

$$
\begin{aligned}
Q_{f}\left(x_{1}, \ldots, x_{n}\right) & =\sum_{i=1}^{n} \sum_{j=1}^{n} h_{i, j} x_{i} x_{j}=\sum_{i=1}^{n} \sum_{j=1}^{n} m_{i+j-2} x_{i} x_{j} \\
& =\sum_{i=1}^{n} \sum_{j=1}^{n} \sum_{\ell=1}^{n} \lambda_{\ell}^{i+j-2} x_{i} x_{j}=\sum_{\ell=1}^{n}\left(\sum_{i=1}^{n} x_{i} \lambda_{\ell}^{i-1}\right)\left(\sum_{j=1}^{n} x_{j} \lambda_{\ell}^{j-1}\right) \\
& =\sum_{\ell=1}^{n}\left(\sum_{j=1}^{n} x_{j} \lambda_{\ell}^{j-1}\right)^{2}=\sum_{\ell=1}^{n} p\left(\lambda_{\ell}\right)^{2}
\end{aligned}
$$

where

$$
p(t)=\sum_{j=1}^{n} x_{j} t^{j-1} \in \mathbf{R}[t]
$$

If $f(x)$ is real-rooted, then $\lambda_{\ell} \in \mathbf{R}$ for all $\ell \in\{1, \ldots, n\}$, and so the sum $p\left(\lambda_{\ell}\right)=$ $\sum_{j=1}^{n} x_{j} \lambda_{\ell}^{j-1}$ is real and $p\left(\lambda_{\ell}\right)^{2} \geq 0$. Thus, if $f(x)$ is real-rooted, then $Q_{f}\left(x_{1}, \ldots, x_{n}\right) \geq$ 0 and the quadratic form $Q_{f}$ is positive semidefinite.

Suppose that $f(x)$ is not real-rooted. In this case, the set $\Lambda$ contains a complex number that is not real, and $\Lambda$ also contains its complex conjugate. We can assume that $\lambda_{1} \in \Lambda$ is not real and that $\lambda_{2}=\overline{\lambda_{1}}$. Note that $\mu_{1} \geq 1$ is the multiplicity of the root $\lambda_{1}$, and that $\mu_{1}$ is also the multiplicity of the root $\lambda_{2}$. By Lemma 2, there exists a polynomial $p(t) \in \mathbf{R}[t]$ of degree $d-1 \leq r-1$ such that

$$
p\left(\lambda_{1}\right)=i, \quad p\left(\lambda_{2}\right)=-i
$$

and

$$
p\left(\lambda_{\ell}\right)=0 \quad \text { for } \ell=3, \ldots, r .
$$

Because $r-1 \leq n-1$, we can write $p(t)=\sum_{j=1}^{n} x_{j} t^{j-1}$, where $\mathbf{x}=\left(\begin{array}{c}x_{1} \\ \vdots \\ x_{n}\end{array}\right) \in \mathbf{R}^{n}$. Note that $x_{j}=0$ for $j=d+1, \ldots, n$. We obtain

$$
\begin{aligned}
Q_{f}\left(x_{1}, \ldots, x_{n}\right) & =\sum_{\ell=1}^{n}\left(\sum_{j=1}^{n} x_{j} \lambda_{\ell}^{j-1}\right)^{2}=\sum_{\ell=1}^{n} p\left(\lambda_{\ell}\right)^{2} \\
& =\mu_{1} p\left(\lambda_{1}\right)^{2}+\mu_{1} p\left(\lambda_{2}\right)^{2}=\mu_{1} i^{2}+\mu_{1}(-i)^{2} \\
& =-2 \mu_{1}<0 .
\end{aligned}
$$

Thus, if $f(x)$ is not real-rooted, then the quadratic form $Q_{f}$ is not positive semidefinite. This completes the proof. 


\section{REFERENCES}

[1] J. Vondrák, Non-constructive methods in combinatorics, Lecture 14, https://theory.stanford.edu/ jvondrak/ MATH233-2016/Math233-lec14.pdf, 2016.

[2] D. Zeilberger, A combinatorial proof of Newton's identities, Discrete Math. 49 (1984), 319.

Department of Mathematics, Lehman College (CUNY), Bronx, NY 10468

E-mail address: melvyn.nathanson@lehman. cuny.edu 